\title{
Long-term Prognostic Value of Homocysteine in Patients with Acute Coronary Syndrome Complicated with Hypertension: A Multicenter Retrospective Study
}

\author{
Qiang Chen \\ Chengdu Third People's Hospital \\ Xunshi Ding \\ Chengdu Third People's Hospital \\ Caiyan Cui \\ Chengdu Third People's Hospital \\ Tao Ye \\ Chengdu Third People's Hospital \\ Lin Cai ( $\nabla$ cailincdsyy@yeah.net) \\ Chengdu Third People's Hospital
}

\section{Research Article \\ Keywords: acute coronary syndrome, homocysteinein, hypertension, prognosis}

Posted Date: November 1st, 2021

DOI: https://doi.org/10.21203/rs.3.rs-1010885/v1

License: (1) This work is licensed under a Creative Commons Attribution 4.0 International License. Read Full License 


\section{Abstract}

Background and aims: This study investigates the long-term prognostic value of homocysteine in patients with acute coronary syndrome complicated with hypertension.

Methods:The current work is a multicenter, retrospective, observational cohort study. We consecutively enrolled 1288 ACS patients hospitalized in 11 general hospitals in Chengdu, China, from June 2015 to December 2019. The patients were divided into hypertension and non-hypertension groups, and each was further classified into hyperhomocysteinemia (H-Hcy) and normal homocysteinemia ( $\mathrm{N}$-Hcy) groups according to the cut-off value of homocysteine predicting long-term mortality during follow-up. In both groups, we used Kaplan-Meier and multivariate Cox regression analysis to assess the relationship between homocysteine and long-term prognosis.

Results: The median follow-up time was 18 months (range: 13.83-22.37). During this period, 78 (6.05\%) death cases were recorded. The hypertension was further divided into H-Hcy $(n=245)$ and N-Hcy $(n=543)$, with an optimal cut-off value of $16.81 \mu \mathrm{mol} / \mathrm{L}$. Similarly, nonhypertension was further divided into H-Hcy $(n=200)$ and N-Hcy $(n=300)$, with an optimal cut-off value of $14 \mu \mathrm{mol} / \mathrm{L}$. Kaplan-Meier survival curves revealed that $\mathrm{H}$-Hcy had a significantly lower survival probability than $\mathrm{N}$-Hcy, both in hypertension and non-hypertension $(\mathrm{P}-$ value<0.01). After adjusting for confounding factors, multivariate Cox regression analysis revealed that $\mathrm{H}-\mathrm{Hcy}(\mathrm{HR}=2.1923,95 \% \mathrm{Cl}: 1.213-$ $3.9625, \mathrm{P} \otimes 0.01$ ) was an independent predictor of long-term all-cause death in ACS with hypertension, but not in non-hypertension.

Conclusion: Elevated homocysteine level predicts risk of all-cause mortality in ACS with hypertension, but not in those without hypertension. it should be considered when determining risk stratification for ACS, particularly those complicating hypertension.

\section{Introduction}

Acute coronary syndrome (ACS) remains a serious type of coronary heart disease (CHD) associated with high morbidity and mortality worldwide [1]. Although percutaneous coronary intervention $(\mathrm{PCl})$ has greatly reduced mortality of these patients, identifying high-risk post$\mathrm{PCl}$ populations and providing them with optimal comprehensive treatment and nursing remains necessary to further improve their prognosis.

Homocysteine (Hcy), derived through methionine (Met) metabolism, has been linked to coronary heart diseases (CHD) [2] by promoting plaque formation and atherosclerosis [3], causing platelet aggregation and blood coagulation, altering lipid metabolism, and triggering inflammatory responses. Previous studies [4-6] demonstrated that elevated Hcy level was independently associated with increased risk of long-term cardiovascular events in patients undergoing PCl. However, the mean Hcy concentrations varied across these studies, and the $\mathrm{H}$ Hcy threshold was inconsistent. As a result, risk stratification based on definite cut values for Hcy may underestimate patients' actual risk.

In addition, the increase in Hcy level is strongly associated with the occurrence and development of hypertension by inhibiting endogenous hydrogen sulfide generation and activating angiotensin-converting enzymes $[7,8]$. Meanwhile, $[9,10]$ reported that hypertension and hyperhomocysteinemia have a significant synergistic effect on cardiovascular disease prognosis. Therefore, Hcy may have a different influence on prognosis in hypertensive and non-hypertensive patients. However, most studies currently use the same homocysteine classification criteria for all ACS patients, regardless of their blood pressure status. Therefore, the study intends to use different cut-off values in hypertensive and non-hypertensive patients to explore the predictive value of Hcy in the long-term prognosis of ACS patients undergoing $\mathrm{PCl}$.

\section{Material And Methods}

\section{Study population and design}

This work is a multicenter, retrospective, observational cohort study. We consecutively enrolled ACS patients who underwent percutaneous coronary therapy (PCl) hospitalized in 11 general medical centers in Chengdu, China, from June 2015 to December 2019. The diagnosis of ACS, including ST-elevation myocardial infarction (STEMI), non-ST elevation myocardial infarction (NSTEMI), and unstable angina pectoris (UA), is based on the corresponding guidelines [11, 12]. The exclusion criteria are as follows: 1) baseline data is unavailable; 2) loss of follow-up; 3) patients with shorter life expectancy complicating with severe chronic lung disease, cardiomyopathy, liver dysfunction, terminal tumor, etc.; and 4) patients died in hospital.

The demographic, clinical, biochemical, and angiographic data were collected by trained professionals from the hospital medical records system. Patients were classified into two groups based on their discharge diagnosis: hypertension and non-hypertension. To increase the prognostic importance of the study further, the optimal cut-off value of plasma homocysteine concentration to measure long-term mortality 
was assessed by receiver operating characteristic (ROC) curve analysis. According to the cut-off value of each group, hypertension was further divided into hyperhomocysteinemia (H-Hcy) $(n=245)$ and normal homocysteinemia(N-Hcy) groups ( $n=543)$ (the area under ROC curve was 0.639 , the sensitivity was $58.8 \%$, the specificity was $71.0 \%$, and the optimal cut-off value was $16.81 \mu \mathrm{mol} / \mathrm{L}$, P-value $<0.001$, Figure 1). Similarly, non-hypertension was subdivided into $\mathrm{H}-\mathrm{Hcy}(\mathrm{n}=200)$ and $\mathrm{N}-\mathrm{Hcy}$ groups $(\mathrm{n}=300)$ (the area under ROC curve was 0.692 , the sensitivity was $77.8 \%$, the specificity was $62.2 \%$, and the optimal cut-off value was $14 \mu \mathrm{mol} / \mathrm{L}, \mathrm{P}$-value $<0.001$, Figure 2 ).

The study is registered in the Chinese Clinical Trials Registry in China (ChiCTR1900025138). The local ethics committee approved the study. Due to the study's retrospective nature, the Committee waived the requirement for formal informed consent.

\section{Follow-up}

After discharge, patients were followed up by a professional cardiologist at 1, 6, and 12 months, and then annually. Prognostic information was obtained by consulting electronic medical records or telephone inquiries. We discontinued follow-up when death was recorded. The primary endpoint was all-cause mortality and cardiovascular mortality. The second primary endpoint includes non-fatal myocardial infarction, revascularization, and non-fatal stroke.

\section{Definitions}

Hypertension was defined as a systolic blood pressure $\geq 140 \mathrm{mmHg}$ and/or a diastolic blood pressure $\geq 90 \mathrm{mmHg}$ during hospitalization or a previous diagnosis of hypertension [13]. Premature acute coronary disease (ACS) refers to the occurrence of ACS in men who are less than 55 years old and women who are less than 65 years old [14]. Multivessel disease [15] was defined as stenosis $₫ 50 \%$ in $₫ 1$ of major coronary arteries.

Cardiovascular mortality [16] was defined as death due to acute myocardial infarction, heart failure, and/or arrhythmia, and unexplained sudden death. Revascularization [17] was defined as the revascularization of any lesion, including percutaneous coronary intervention (PCI) or coronary artery bypass grafting (CABG). Stroke was defined as ischemic or hemorrhagic stroke during the follow-up period confirmed by imaging and diagnosed by professional neurologists.

\section{Statistical analysis}

Continuous data are expressed as mean \pm SD or inter-quartiles range (IQR). They were compared using Student's t-test or Mann-Whitney $U$ test, as appropriate. Categorical variables are expressed as percentages. The comparisons between categorical variables were performed using 2 test or Fisher exact test. The optimal cut-off value for serum homocysteine was obtained from ROC curve analysis. The time-toevent data were plotted using Kaplan-Meier method, and a log-rank test was used to evaluate differences between groups. Cox proportional hazards regression modeling was used to assess whether elevated homocysteine concentration was associated with a worse long-term prognosis. MedCalc Statistical Software version 19.6.1 (MedCalc Software, Ostend, Belgium) was used for all statistical analyses. All statistical tests were 2-tailed, and a p-value $<0.05$ was considered to be statistically significant.

\section{Results}

\section{Baseline characteristics of patients}

This study included 1288 ACS patients (595 UA, 396 STEMI, and 297 NSTEMI), with 788 hypertension patients (61.18\%). The median plasma homocysteine level was 13.82 (25th to 75th centiles, $11.04-18.44$ ) mmol/L in hypertension group and 12.85 (25th to 75th centiles, 10.4-16.5) $\mathrm{mmol} / \mathrm{L}$ in non-hypertension group. Table 1 summarizes baseline demographic, clinical, biochemical, and angiographic data of hypertension and non-hypertension stratified by cut-off value for plasma homocysteine.

Table 1

Baseline characteristics 


\begin{tabular}{|c|c|c|c|c|c|c|}
\hline \multirow[t]{2}{*}{ Characteristics } & \multicolumn{3}{|c|}{ Hypertension囚n=788ם } & \multicolumn{3}{|c|}{ Non-hypertension囚n=500] } \\
\hline & $H-H c y \rrbracket n=245 \square$ & $\mathrm{N}-\mathrm{Hcy} 囚 \mathrm{n}=543 \square$ & $t / c 2 \bigotimes P]$ & H-Hcy $(n=200)$ & $\mathrm{N}-\mathrm{Hcy}(\mathrm{n}=300)$ & $\mathrm{t} / \mathrm{c} 2 \bigotimes \mathrm{P} \rrbracket$ \\
\hline Age (years) & $70.82 \pm 11.08$ & $67.82 \pm 11.08$ & 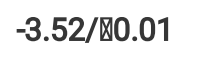 & $65.52 \pm 14.53$ & $61.6 \pm 11.23$ & 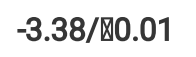 \\
\hline Male (\%) & 19凶77.6\%ם & $313 \otimes 60.9 \% \square$ & 20.75/ख0.01 & 169『84.5\%ם & $240(80 \%)$ & $1.63 / 0.2$ \\
\hline Smoking(\%) & 78区31.8\%[ & $176 \rrbracket 32.4 \% \square$ & $0.026 / 0.873$ & $92 \llbracket 46 \% \square$ & 146ه48.7\%ם & $0.34 / 0.56$ \\
\hline $\begin{array}{l}\text { previous } \\
\text { Revascularization } \\
\text { therapy(\%) }\end{array}$ & $24 \llbracket 9.8 \% \square$ & 47凶8.7\% & $0.268 / 0.605$ & 19凶9.5\%马 & $22 \llbracket 7.3 \% \square$ & $0.748 / 0.387$ \\
\hline Previous stroke(\%) & $29 \llbracket 11.8 \% \square$ & $30(5.5 \%)$ & 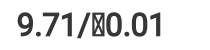 & $10 \rrbracket 5 \% \square$ & $3 \otimes 1 \% \square$ & 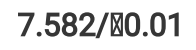 \\
\hline Diabetes mellitus $₫ \%$ & $92(37.6 \%)$ & $198(36.5 \%)$ & $0.086 / 0.77$ & $35 \llbracket 17.5 \% \square$ & $81(27 \%)$ & $6.08 / 0.014$ \\
\hline BNP囚pg/mLם & $175.2 \otimes 78 \bigotimes 577.10$ & $\begin{array}{l}120.4 \llbracket 59.2 \square \\
378.5 \square\end{array}$ & 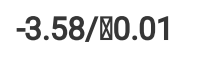 & $\begin{array}{l}170.85 \times 68 \square \\
435.49 \square\end{array}$ & $\begin{array}{l}130.9 \otimes 59.25 \square \\
365.45 \square\end{array}$ & $-2.199 / 0.028$ \\
\hline $\begin{array}{l}\text { Serum } \\
\text { creatinine }(\mu \mathrm{mol} / \mathrm{L})\end{array}$ & $96(80.15,126.5)$ & $74.7(62.9,87.6)$ & $\begin{array}{l}-11.743 / \square \\
0.01\end{array}$ & $79.95(68.27,95.5)$ & $70.9(62.37,81.47)$ & 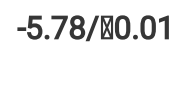 \\
\hline Triglyceride(mmol/L) & $1.41(1.01,2.13)$ & $1.45(1.04,2.2)$ & $-0.919 / 0.358$ & $1.33(0.97,1.99)$ & $1.38(1,1.97)$ & $-0.16 / 0.873$ \\
\hline $\begin{array}{l}\text { Total cholesterol } \\
(\mathrm{mmol} / \mathrm{L})\end{array}$ & $4.3(3.51,5.11)$ & $4.32(3.6,5.23)$ & $-0.989 / 0.323$ & $4.29(3.51,4.99)$ & $4.47(3.84,5.2)$ & $-2.216 / 0.027$ \\
\hline LDL-C(mmol/L) & $2.44(1.94,3.08)$ & $2.63(2.06,3.34)$ & $-2.453 / 0.014$ & $2.65(2.1,3.25)$ & $2.76(2.24,3.34)$ & $-1.627 / 0.14$ \\
\hline HDL-C(mmol/L & $1.09(0.9,1.31)$ & $1.14(0.97,1.37)$ & $-2.762 /$ /ख0.01 & $1.11(0.94,1.35)$ & $1.1(0.94,1.32)$ & $-0.608 / 0.543$ \\
\hline $\operatorname{Lp}(a)(m g / L)$ & $133(60.1,265.52)$ & $144(67.9,273.3)$ & $-0.833 / 0.405$ & $\begin{array}{l}189.6 ه 76.9 \square \\
266.55 \square\end{array}$ & $\begin{array}{l}163.35 \square 65.77 \square \\
327.75 \square\end{array}$ & $-0.462 / 0.644$ \\
\hline $\begin{array}{l}\text { Multivessel } \\
\text { disease(\%) }\end{array}$ & $150(61.2 \%)$ & $319(58.7 \%)$ & $0.43 / 0.512$ & $110(55 \%)$ & $153(51 \%)$ & $0.77 / 0.38$ \\
\hline ejection fraction & $53.44 \pm 10.17$ & $56.06 \pm 8.99$ & 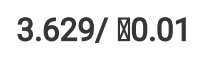 & $53.45 \pm 9.77$ & $56.12 \pm 9.33$ & $3.078 / \mathbb{\mathbb { X } 0 . 0 1}$ \\
\hline $\mathrm{EF} \otimes 40(\%)$ & 28囚11.4\%[ & $32 \varangle 5.9 \% \square$ & 7.354/ख0.01 & $17 \llbracket 8.5 \% \square$ & 19ष6.3\%[ & $0.843 / 0.359$ \\
\hline calcified lesions $\llbracket \% \square$ & $36(14.7 \%)$ & $70(12.9 \%)$ & $0.471 / 0.492$ & $19(9.5 \%)$ & $13(4.3 \%)$ & $5.347 / 0.021$ \\
\hline Premature ACS(\%) & $29(11.8 \%)$ & $115(21.2 \%)$ & $9.865 /$ ख0.01 & $49 \llbracket 24.5 \% \square$ & $98 \times 32.7 \% \square$ & $3.856 / 0.05$ \\
\hline ACS $₫ \square \square$ & & & $4.528 / 0.104$ & & & $1.322 / 0.516$ \\
\hline UA & $112 \otimes 45.7 \% \square$ & $287(52.9 \%)$ & & $75 \llbracket 37.5 \% \square$ & $121 \llbracket 40.3 \% \square$ & \\
\hline NSTEMI & $68 \llbracket 27.8 \% \square$ & $117(21.5 \%)$ & & $50 \times 25 \% \square$ & $62 \llbracket 20.7 \% \square$ & \\
\hline STEMI & $65(26.5 \%)$ & $139(25.6 \%)$ & & $75 \llbracket 37.5 \% \square$ & $117 \rrbracket 39 \% \square$ & \\
\hline
\end{tabular}

Hyperhomocysteinemia patients were older and complicated with a higher level of serum BNP, creatinine, and lower ejection fraction in hypertension and non-hypertension ( $\mathrm{p}$-value $<0.05$ for both). In addition, in terms of medical history, the proportion of complications with previous stroke was higher in the population with $\mathrm{H}$-Hcy in both groups ( $\mathrm{p}$-value $<0.05$ for both). In the hypertension group, $\mathrm{H}$-Hcy group patients had more males and lower LDL-C and HDL-C levels ( $p$-value < 0.05 ) than the N-Hcy group. Moreover, the proportion of heart failure ( $\mathrm{EF}<40 \%)$ was higher in patients with $\mathrm{H}-\mathrm{Hcy}(\mathrm{P} \otimes 0.05)$. In non-hypertension group, $\mathrm{H}$-Hcy patients had a higher proportion of calcified coronary lesions, and these patients had lower levels of total cholesterol ( $p$-value $<0.05)$. Additionally, complicating smoking, previous revascularization therapy, diabetes mellitus, multivessel disease, triglyceride, and $L p(a)$ levels did not differ between hypertension and nonhypertension subgroups ( $p$-value $>0.05$ ).

\section{Long-term clinical outcomes}

The median follow-up time was 18 (range: 13.83-22.37) months. During this period, 78 (6.05\%), $34(2.64 \%), 104(8.07 \%)$, and $10(0.77 \%)$ cases of death, non-fatal MI, revascularization, and non-fatal stroke were recorded, respectively. The number of all-cause mortality and cardiac death events in patients with $\mathrm{H}$-Hcy was higher than in those with normal homocysteine levels in hypertension and non- 
hypertension groups ( $\mathrm{p}$-value『0.01) (Table 2). Kaplan-Meier survival curves revealed that $\mathrm{H}$-Hcy group had a significantly lower survival probability than N-Hcy group, both in hypertension (Figure 3A and B) and non-hypertension (Figure 4A and B) groups (p-value < 0.01 ).

Table 2

Long-term clinical outcomes

\begin{tabular}{|c|c|c|c|c|c|c|}
\hline & \multicolumn{3}{|c|}{ Hypertension $₫ n=788 \square$} & \multicolumn{3}{|c|}{ Non-hypertension $囚 \mathrm{n}=500 \square$} \\
\hline & $\mathrm{H}-\mathrm{Hcy} 囚 n=245 \square$ & $\mathrm{N}-\mathrm{Hcy} 囚 \mathrm{n}=543 \square$ & $\mathrm{t} / \mathrm{c} 2 \otimes P \square$ & $\mathrm{H}-\mathrm{Hcy}(\mathrm{n}=200)$ & $\mathrm{N}-\mathrm{Hcy}(\mathrm{n}=300)$ & $\mathrm{t} / \mathrm{c} 2 \bigotimes \mathrm{PQ}$ \\
\hline All-cause death & $30 \bigotimes 12.2 \%$ & $21 \rrbracket 3.9 \% \square$ & 19.57/ख0.01 & $21 \otimes 10.5 \% \square$ & $6 \llbracket 2.0 \%$ & 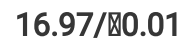 \\
\hline Cardiac death & $23 \varangle 9.4 \% \square$ & $17 \rrbracket 3.1 \% \square$ & 13.72/ख0.01 & $16 \llbracket 8 \%)$ & $3(1 \%)$ & 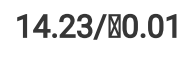 \\
\hline Non-fatal MI & $8(3.3 \%)$ & $12(2.2 \%)$ & $0.76 / 0.383$ & $7 \rrbracket 3.5 \% \square$ & $7 \llbracket 2.3 \% \square$ & $0.6 / 0.439$ \\
\hline Revascularization & $22 \times 9 \%)$ & $43(7.9 \%)$ & $0.251 / 0.616$ & $13(6.5 \%)$ & $26(8.7 \%)$ & $0.783 / 0.376$ \\
\hline Non-fatal stroke & $3 \otimes 1.2 \% \square$ & 4囚0.7\% & $0.07 / 0.791$ & 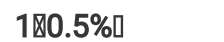 & $2 \times 0.7 \% \square$ & $\bowtie 0.99$ \\
\hline
\end{tabular}

\section{Predictors for long-term all-cause mortality}

Cox regression analysis for non-hypertension revealed that independent predictors of all-cause death was age (HR=1.0618, $95 \% \mathrm{Cl}$ : 1.0225 1.1026, $\mathrm{P} \otimes 0.01$ ), and the protective factor was ejection fraction (HR=0.9387, 95\% Cl: 0.9023-0.9765, P凶0.01) (Table 3). However, for hypertension, Cox regression analysis revealed that independent predictors of all-cause death were age ( $\mathrm{HR}=1.055,95 \% \mathrm{Cl}$ : $1.021-1.0902, \mathrm{P} \otimes$ 0.01), H-Hcy (HR=2.1923, 95\% Cl: 1.213-3.9625, Pख0.01) and serum creatinine (HR=1.018, 95\% Cl: 1.0003-1.0032, $\mathrm{P} \otimes 0.01)$, and the protective factor also was ejection fraction (HR=0.9505, 95\% Cl: 0.9279-0.9737, P凶0.01) (Table4)..

Table 3

Non-hypertension

\begin{tabular}{|lllll|}
\hline Covariate & $\beta$-value & P-value & HR & $95 \%$ Cl of HR \\
\hline H-Hcy & 0.9678 & 0.0521 & 2.6322 & 0.9914 to 6.9889 \\
\hline Age & 0.05996 & 0.0018 & 1.0618 & 1.0225 to 1.1026 \\
\hline Gender(female) & 0.4657 & 0.3165 & 1.5932 & 0.6405 to 3.9628 \\
\hline Serum creatinine & 0.007085 & 0.1606 & 1.0071 & 0.9972 to 1.0171 \\
\hline Ejection fraction $\varangle \mathrm{EF} \otimes$ & -0.06329 & 0.0017 & 0.9387 & 0.9023 to 0.9765 \\
\hline
\end{tabular}

Table 4

Hypertension

\begin{tabular}{|lllll|}
\hline Covariate & $\beta$-value & P-value & HR & $95 \%$ Cl of HR \\
\hline H-Hcy & 0.7850 & 0.0093 & 2.1923 & 1.2130 to 3.9625 \\
\hline Age & 0.05355 & 0.0014 & 1.0550 & 1.0210 to 1.0902 \\
\hline Gender(female) & 0.3958 & 0.1856 & 1.4855 & 0.8268 to 2.6691 \\
\hline Serum creatinine & 0.001765 & 0.0186 & 1.0018 & 1.0003 to 1.0032 \\
\hline Ejection fraction $₫ \mathrm{EF} \otimes$ & -0.05075 & $<0.0001$ & 0.9505 & 0.9279 to 0.9737 \\
\hline
\end{tabular}

\section{Discussion}


Elevated serum homocysteine has been reported to be an independent risk factor for cardiovascular and cerebrovascular diseases [6, 18 , 19]. However, disputes [20] still exist regarding whether elevated homocysteine levels have predictive value for long-term prognosis in ACS patients. Therefore, this study sought to determine the relationship between homocysteine and long-term outcome in ACS patients undergoing $\mathrm{PCl}$. The results unveiled that $\mathrm{H}$-Hcy patients have higher all-cause mortality and cardiac death events than those without $\mathrm{H}-\mathrm{Hcy}$ in ACS, regardless of hypertension. On the other hand, elevated serum homocysteine concentration is an independent predictor for long-term all-cause mortality after discharge in ACS patients with hypertension but not in ACS patients without hypertension.

Homocysteine is associated with plaque formation and atherosclerosis progression [3] by damaging vascular endothelial cells, altering lipid metabolism, and triggering inflammatory responses. In addition, it may be involved in acute coronary events by disrupting the balance between blood coagulation and fibrinolysis, leading to platelet aggregation and blood coagulation [21]. Thus, homocysteine has been identified as a prognostic factor for coronary heart disease. The study of $\mathrm{Li}, \mathrm{S}$ et al. [22] revealed that $\mathrm{H}-\mathrm{Hcy}(\mathrm{HR}=1.075,95 \% \mathrm{Cl}$ : 1.032-1.120, $\mathrm{P} \otimes 0.01)$ is an independent predictor of MACCE in patients with coronary heart disease (CHD) who underwent drug-eluting stent implantation. Moreover, a meta-analysis [6] of 19 cohort studies (4340 patients) revealed that elevated Hcy levels increased the risk of all-cause mortality by an average of 3.19-fold ( $\mathrm{HR}=3.19,95 \% \mathrm{Cl}$ : 1.90-5.34, P凶0.01), MACE by 1.51-fold (HR = 1.51, 95\% Cl: 1.23-1.85, Pख0.01), and cardiac death by 2.76 -fold $(\mathrm{HR}=2.76,95 \% \mathrm{Cl}$ : 1.44-5.32, $\mathrm{P} \otimes 0.01)$ in post-PCl patient. However, genetic background, eating habits, and living habits all affected the serum level of Hcy [23]. The mean Hcy levels varied between different studies. Simultaneously, the threshold for H-Hcy was inconsistent among various studies [22, 24]. Therefore, using definite cut-off values of Hcy concentration defined by guidelines or previous classical studies for risk stratification may underestimate the actual risk of patients. In addition, because homocysteine and hypertension have a synergistic effect on the prognosis of cardiovascular disease [9], homocysteine may have different effects on prognosis of ACS patients with complicated hypertension or not. As a result, we divided ACS patients into hypertension and non-hypertension groups and used ROC analysis to determine the optimal critical value of homocysteine for predicting long-term death in ACS patients in each of the two groups. The two groups were then subdivided into two subgroups based on the optimal cut-off value: an $\mathrm{H}-\mathrm{Hcy}$ group and a normal Hcy group. As far as we know, the research method we adopted was more scientific and reasonable than those previously used.

In our study, Kaplan-Meier survival curves revealed that H-Hcy was associated with long-term mortality, including all-cause mortality and cardiac death, during 18-month median follow-up period in two groups, consistent with previous studies [4, 6]. Meanwhile, we observed that patients in the H-Hcy group were older and complicated with higher levels of serum BNP, creatinine, and uric acid levels and lower ejection fraction. These factors are variables of GRACE risk score, which is closely associated with the prognosis of ACS patients. Aslihan Calim et al. [5] recently reported a significant positive correlation between homocysteine and GRACE risk scores in ACS patients. Although controversies exist [25], most observational studies demonstrated that homocysteine is independent of other classic risk factors for cardiovascular diseases.

After adjusting for other risk factors, including $\mathrm{H}-\mathrm{Hcy}$, age, gender, serum creatinine, and ejection fraction, multivariate Cox regression analyses revealed that $\mathrm{H}$-Hcy was strongly associated with long-term all-cause mortality in hypertension, but not in non-hypertension. Numerous possible explanations have been proposed, including the following. First, hypertension and homocysteine may have a synergistic effect on the prognosis of ACS. On the one hand, homocysteine plays a key role in atherosclerosis and plaque formation, strongly associated with long-term adverse events. On the other hand, ACS patients with hypertension have a poor long-term prognosis. Homocysteine is closely associated with hypertension development by activating angiotensin-converting enzyme. Thus, when hyperhomocysteine and hypertension are combined, the effect of " $1+1>2$ " may occur. Second, the proportion of patients with heart failure (EF $₫ 4 \%$ ) was higher in $\mathrm{H}$-Hcy patients in the hypertension group, which is significantly linked to long-term prognosis of patients.

Furthermore, we found that the proportion of complicated strokes was higher in $\mathrm{H}-\mathrm{Hcy}$ group than in N-Hcy group, consistent with the classical theory [26]. A case-control study conducted in six centers in China revealed that the risk of stroke in high Hcy population increased by $87 \%$ [27]. However, the study we conducted failed to establish a link between homocysteine and non-fatal stroke events in the long-term follow-up. This is due to the small sample size, short follow-up time, and few endpoints observed in this study.

However, our investigation has several limitations. First, this study only discussed the relationship between Hcy levels and long-term prognosis of ACS patients. There has been no discussion of whether lowering Hcy levels can improve the long-term prognosis of ACS patients, requiring additional research. Second, this was a retrospective study with relatively small sample size and relatively shorter followup duration, introducing bias. Third, there are differences in comprehensive management and treatment ability of ACS patients in different hospitals, which may influence observation results. Additionally, there may be discrepancies in the Hcy detection methods used in different hospitals. Finally, this paper did not discuss the impact of combined medication, including ACEI/ARB, antiplatelet drugs, statins, and $\beta$ receptor antagonists, on long-term cardiovascular events.

\section{Conclusion}


This paper suggests that elevated homocysteine levels independently predict all-cause mortality risk in ACS patients with hypertension but not in those without hypertension. Thus, independent risk factors for cardiovascular events should be considered when evaluating risk stratification for ACS patients. Despite this, additional perspective, large sample studies are required to further evaluate the relationship between elevated homocysteine levels and cardiovascular events.

\section{Declarations}

\section{Ethics approval and consent to participate}

The study was registered in the Chinese Clinical Trials Registry in China (ChiCTR1900025138). The study was approved by Ethics Committee of Chengdu Third People's Hospital. Due to the study's retrospective nature, the Committee waived the requirement for formal informed consent.

We stated that our protocol was performed in accordance with the relevant guidelines and the Declaration of Helsinki.

\section{Consent for publication}

Not applicable

\section{Availability of data and material}

The datasets used or analysed during the current study are available from the corresponding author on reasonable request.

\section{Competing interests}

The authors declare no conflict of interest.

\section{Funding}

This research was supported by the Science and Technology Department of Sichuan, China(grant number 2021YJ0215).

Sponsor's Role:None

\section{Authors' contributions}

Lin Cai performed the experiment;

Xunshi Ding contributed significantly to analysis and manuscript preparation;

Qiang Chen performed the data analyses and wrote the manuscript;

Caiyan Cui and Tao Ye helped perform the collection of data;

All authors reviewed the manuscript

\section{Acknowledgements}

The authors would like to thank the Science and Technology Department of Sichuan, China for their support

\section{References}

1. Piironen M, Ukkola O, Huikuri H, Havulinna AS, Koukkunen H, Mustonen J, Ketonen M, Lehto S, Airaksinen J, Antero Kes?niemi Y, Salomaa V. Trends in long-term prognosis after acute coronary syndrome. Eur J Prev Cardiol 2017; 24:274-80. 
2. Troughton JA, Woodside JV, Young IS, Arveiler D, Amouyel P, Ferri “.'res J, Ducimeti”.'re P, Patterson CC, Kee F, Yarnell JW, Evans A, Group PS. Homocysteine and coronary heart disease risk in the PRIME study. Atherosclerosis 2007; 191:90-7.

3. McCully KS. Homocysteine and the pathogenesis of atherosclerosis. Expert Rev Clin Pharmacol 2015; 8:211-9.

4. Zhu M, Mao M, Lou X. Elevated homocysteine level and prognosis in patients with acute coronary syndrome: a meta-analysis. Biomarkers 2019; 24:309-16.

5. Calim A, Turkoz FP, Ozturkmen YA, Mazi EE, Cetin EG, Demir N, Borlu F. The Relation between Homocysteine Levels in Patients with Acute Coronary Syndrome and Grace Score. 2020; 54:346-50.

6. Zhang Z, Xiao S, Yang C, Ye R, Hu X, Chen X. Association of Elevated Plasma Homocysteine Level with Restenosis and Clinical Outcomes After Percuta neous Coronary Interventions: a Systemic Review and Meta-analysis. Cardiovasc Drugs Ther 2019; 33:353-61.

7. Fu L, Li YN, Luo D, Deng S, Wu B, Hu YQ. Evidence on the causal link between homocysteine and hypertension from a meta-analysis of $40 ? 173$ indi viduals implementing Mendelian randomization. J Clin Hypertens (Greenwich) 2019; 21:1879-94.

8. Onyemelukwe OU, Maiha BB. Prevalence of hyperhomocysteinaemia, selected determinants and relation to hypertension severity in $\mathrm{N}$ orthern-Nigerian hypertensives: the ABU homocysteine survey. Ghana Med J 2020; 54:17-29.

9. Liu M, Fan F, Liu B, Jia J, Jiang Y, Sun P, He D, Liu J, Li Y, Huo Y, Li J, Zhang Y. Joint Effects of Plasma Homocysteine Concentration and Traditional Cardiovascular Risk Factors on the Risk of New-Onset Peripheral Arterial Disease. Diabetes Metab Syndr Obes 2020; 13:3383-93.

10. Li J, Jiang S, Zhang Y, Tang G, Wang Y, Mao G, Li Z, Xu X, Wang B, Huo Y. H-type hypertension and risk of stroke in chinese adults: A prospective, nested case-control study. J Transl Int Med 2015; 3:171-8.

11. Arslan F, Bongartz L, Ten Berg JM, Jukema JW, Appelman Y, Liem AH, de Winter RJ, van 't Hof AWJ, Damman P. 2017 ESC guidelines for the management of acute myocardial infarction in patients presenting with ST- segment elevation: comments from the Dutch ACS working group. Neth Heart J 2018; 26:417-21.

12. Collet JP, Thiele H, Barbato E, Barth“" "“|'Imy O, Bauersachs J, Bhatt DL, Dendale P, Dorobantu M, Edvardsen T, Folliguet T, Gale CP, Gilard M, Jobs A, J“1ni P, Lambrinou E, Lewis BS, Mehilli J, Meliga E, Merkely B, Mueller C, Roffi M, Rutten FH, Sibbing D, Siontis GCM, Group ESCSD. 2020 ESC Guidelines for the management of acute coronary syndromes in patients presenting without per sistent ST-segment elevation. Eur Heart J 2021; 42:1289-367.

13. Force USPST, Krist AH, Davidson KW, Mangione CM, Cabana M, Caughey AB, Davis EM, Donahue KE, Doubeni CA, Kubik M, Li L, Ogedegbe G, Pbert L, Silverstein M, Stevermer J, Tseng CW, Wong JB. Screening for Hypertension in Adults: US Preventive Services Task Force Reaffirmation Recommendation Statement. Jama 2021; 325:1650-6.

14. LaBarbera M, Milechman G, Dulbecco F. Premature coronary artery disease in a patient with glycogen storage disease III. J Invasive Cardiol 2010; 22:E156-8.

15. Ueyama H, Kuno T, Yasumura K, Vengrenyuk Y, Takagi H, Barman N, Suleman J, Banning AS, Boxma-de Klerk BM, Smits PC, Kini A, Sharma SK. Meta-Analysis Comparing Same-Sitting and Staged Percutaneous Coronary Intervention of Non-Culprit Art ery for STElevation Myocardial Infarction with Multivessel Coronary Disease. Am J Cardiol 2021.

16. Benn M, Tybj?rg-Hansen A, Nordestgaard BG. Low LDL Cholesterol by PCSK9 Variation Reduces Cardiovascular Mortality. J Am Coll Cardiol 2019; 73:3102-14.

17. Azadani PN, Miller RJH, Sharir T, Diniz MA, Hu LH, Otaki Y, Gransar H, Liang JX, Eisenberg E, Einstein AJ, Fish MB, Ruddy TD, Kaufmann PA, Sinusas AJ, Miller EJ, Bateman TM, Dorbala S, Di Carli M, Tamarappoo BK, Dey D, Berman DS, Slomka PJ. Impact of Early Revascularization on Major Adverse Cardiovascular Events in?Relation to Automatically Quantified?Ischemia. JACC Cardiovasc Imaging 2021; 14:644-53.

18. Li N, Tian L, Ren J, Li Y, Liu Y. Evaluation of homocysteine in the diagnosis and prognosis of coronary slow flow syndrome. Biomark Med 2019; 13:1439-46.

19. Wang D, Wang W, Wang A, Zhao X. Association of Severity and Prognosis With Elevated Homocysteine Levels in Patients With Intracerebra I Hemorrhage. Front Neurol 2020; 11:571585.

20. Miao L, Deng GX, Yin RX, Nie RJ, Yang S, Wang Y, Li H. No causal effects of plasma homocysteine levels on the risk of coronary heart disease or acute myocar dial infarction: A Mendelian randomization study. Eur J Prev Cardiol 2021; 28:227-34.

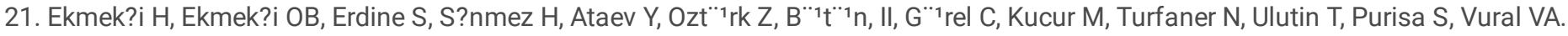
Effects of serum homocysteine and adiponectin levels on platelet aggregation in untreated patients wi th essential hypertension. $J$ Thromb Thrombolysis 2009; 28:418-24.

22. Li S, Sun L, Qi L, Jia Y, Cui Z, Wang Z, Li F, Zhao X. Effect of High Homocysteine Level on the Severity of Coronary Heart Disease and Prognosis After Stent Implantation. J Cardiovasc Pharmacol 2020; 76:101-5.

Page 8/11 
23. Ma T, Sun XH, Yao S, Chen ZK, Zhang JF, Xu WD, Jiang XY, Wang XF. Genetic Variants of Homocysteine Metabolism, Homocysteine, and Frailty - Rugao Longevity and Ageing S tudy. J Nutr Health Aging 2020; 24:198-204.

24. Bickel C, Schnabel RB, Zengin E, Lubos E, Rupprecht H, Lackner K, Proust C, Tregouet D, Blankenberg S, Westermann D, Sinning C. Homocysteine concentration in coronary artery disease: Influence of three common single nucleotide po lymorphisms. Nutr Metab Cardiovasc Dis 2017; 27:168-75.

25. Han L, Wu Q, Wang C, Hao Y, Zhao J, Zhang L, Fan R, Liu Y, Li R, Chen Z, Zhang T, Chen S, Ma J, Liu S, Peng X, Duan S. Homocysteine, Ischemic Stroke, and Coronary Heart Disease in Hypertensive Patients: A Population-Base d, Prospective Cohort Study. Stroke 2015; 46:1777-86.

26. Zhang H, Huang J, Zhou Y, Fan Y. Association of homocysteine level with adverse outcomes in patients with acute ischemic stroke: A met a-analysis. Curr Med Chem 2021

27. Li Z, Sun L, Zhang H, Liao Y, Wang D, Zhao B, Zhu Z, Zhao J, Ma A, Han Y, Wang Y, Shi Y, Ye J, Hui R, Multicenter Case-Control Study in C. Elevated plasma homocysteine was associated with hemorrhagic and ischemic stroke, but methylenetetrah ydrofolate reductase gene C677T polymorphism was a risk factor for thrombotic stroke: a Multicenter Case-Control Study in China. Stroke 2003; 34:2085-90.

\section{Figures}

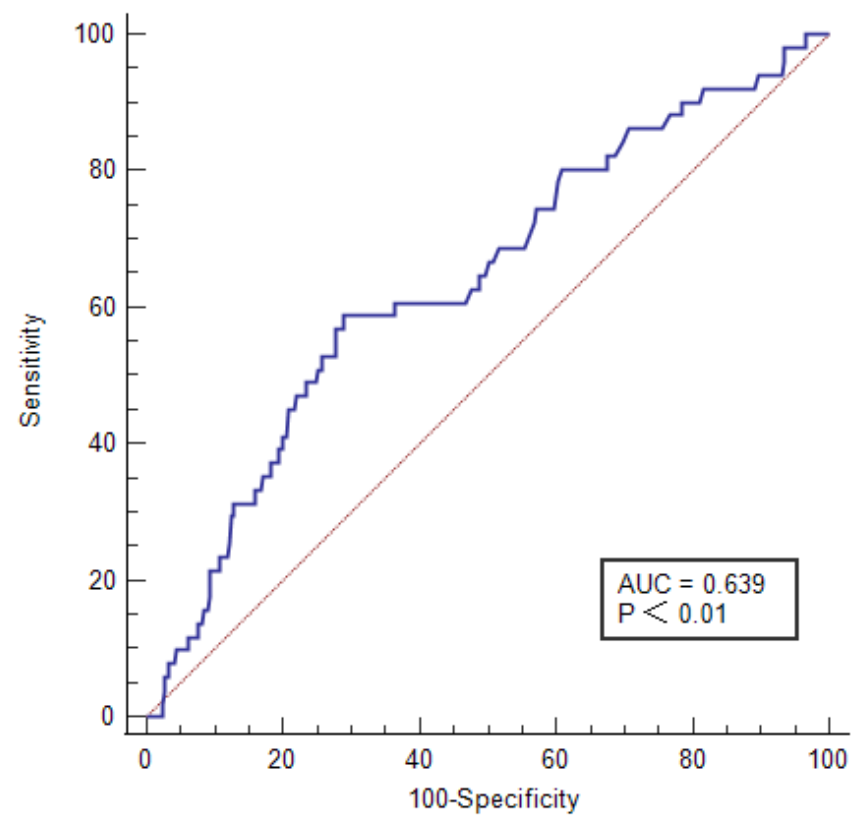

Figure 1

According to the cut-off value of each group, hypertension was further divided into hyperhomocysteinemia $(\mathrm{H}-\mathrm{Hcy})(\mathrm{n}=245)$ and normal homocysteinemia(N-Hcy) groups $(n=543)$ (the area under ROC curve was 0.639 , the sensitivity was $58.8 \%$, the specificity was $71.0 \%$, and the optimal cut-off value was $16.81 \mu \mathrm{mol} / \mathrm{L}, \mathrm{P}$-value $<0.001$, Figure 1). 


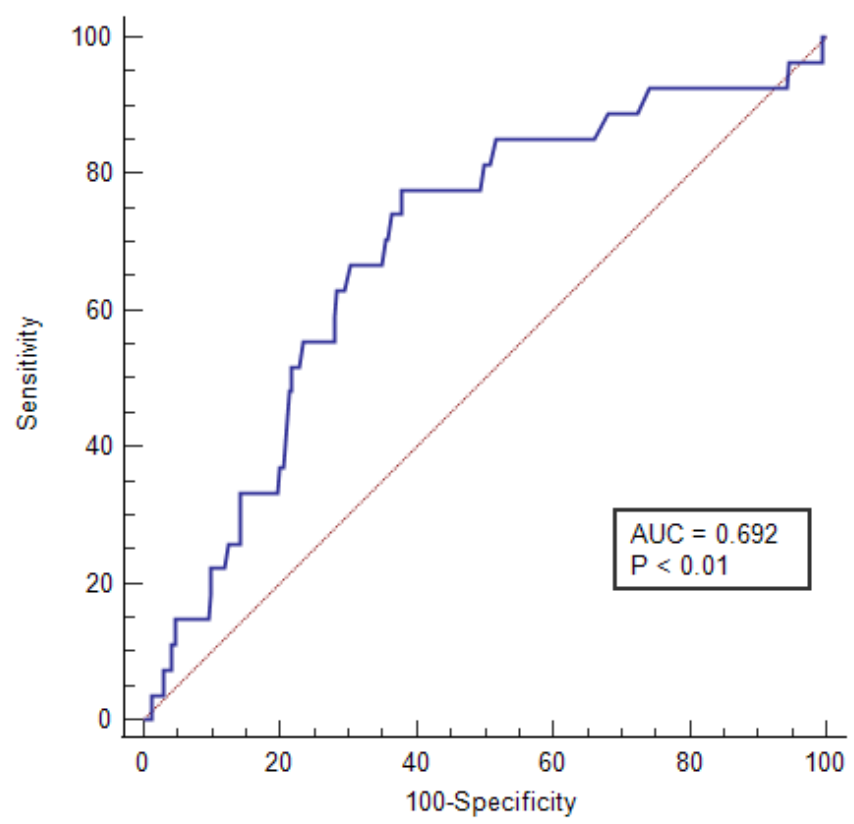

\section{Figure 2}

non-hypertension was subdivided into H-Hcy $(n=200)$ and $\mathrm{N}$-Hcy groups $(n=300)$ (the area under ROC curve was 0.692 , the sensitivity was $77.8 \%$, the specificity was $62.2 \%$, and the optimal cut-off value was $14 \mu \mathrm{mol} / \mathrm{L}$, P-value $<0.001$, Figure 2 ).

\section{Hypertension Figure3}

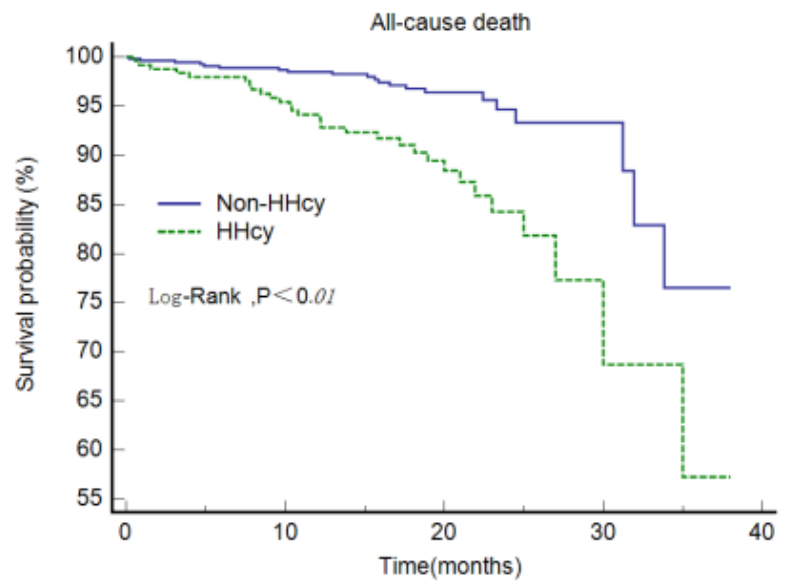

A

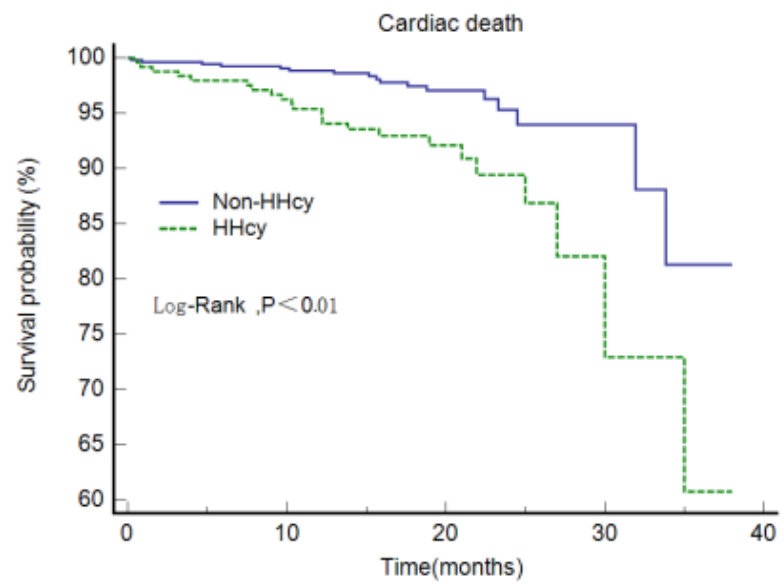

B

\section{Figure 3}

Kaplan-Meier survival curves revealed that $\mathrm{H}$-Hcy group had a significantly lower survival probability than $\mathrm{N}$-Hcy group, both in hypertension (Figure $3 \mathrm{~A}$ and $\mathrm{B}$ ) 


\section{Non-hypertension $\quad$ Figure4}

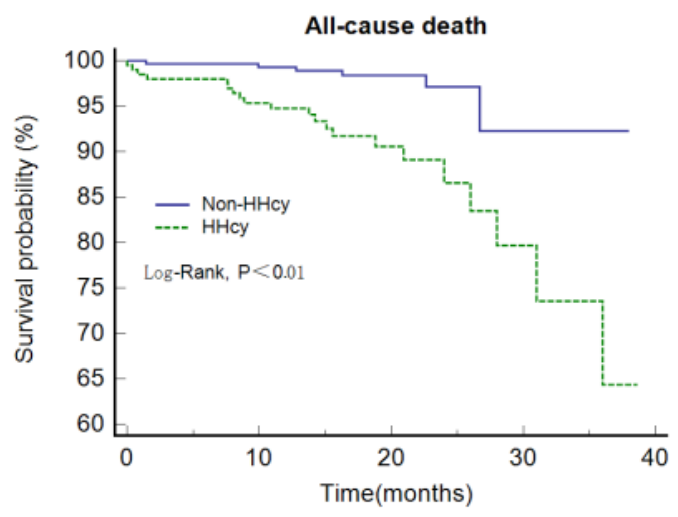

A

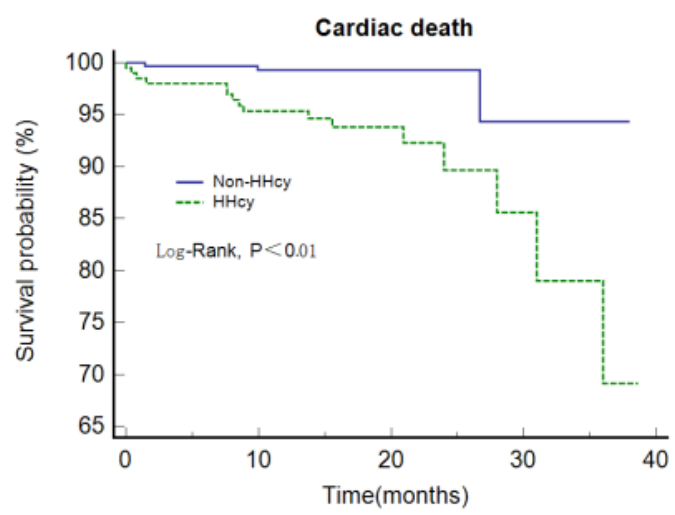

B

Figure 4

non-hypertension (Figure 4A and B) groups ( $p$-value $<0.01)$. 